\title{
Nota científica \\ EFECTO DE BUPROFEZIN, PYRIPROXIFEN Y ACEITE MINERAL EN POSTCOSECHA SOBRE ESCAMA DE SAN JOSÉ (Diaspidiotus perniciosus Comstock) EN MANZANO
}

\author{
Short communication \\ EFFECTS OF POST-HARVEST APPLICATIONS OF BUPROFEZIN, \\ PYRIPROXYFEN AND MINERAL OIL AGAINST SAN JOSE SCALE \\ (Diaspidiotus perniciosus Comstock) ON APPLE TREES \\ Luis Sazo ${ }^{1^{*}}$, Eugenio Asiain ${ }^{1}$, Hugo Sepulveda ${ }^{1}$, y Mayerly Prieto $^{1}$ \\ ${ }^{1}$ Universidad de Chile, Facultad de Ciencias Agronómicas. Casilla 1004, Santiago de Chile. Chile. \\ *Autor para correspondencia E-mail: 1sazo@uchile.cl
}

\section{RESUMEN}

Diaspidiotus perniciosus es una plaga importante de frutales de carozo y pomáceas que puede ser controlada mediante aplicaciones de postcosecha. El objetivo de este estudio fue determinar el efecto de pyriproxyfen y buprofezin sobre los estados juveniles (gorrita negra y gorrita gris) de $D$. perniciosus, en manzanas var. Royal Gala. El estudio se realizó en otoño del 2012 con aplicaciones de insecticidas solos y en mezcla con aceite mineral al $2 \%$ y un testigo aplicado con agua. Las aplicaciones se realizaron con pitón, 250 PSI y $3500 \mathrm{~L} \mathrm{ha}^{-1}$, sobre árboles de manzano infestados. La evaluación se realizó bajo lupa estereoscópica en laboratorio, 48 días después de la aplicación (DDA) sobre juveniles fijados en ramillas de la temporada. Los resultados se expresaron en porcentaje de mortalidad. Se empleó un diseño completamente al azar con 6 tratamientos y 4 repeticiones; la unidad experimental fueron 2 árboles contiguos de los cuales se colectó una muestra compuesta de 30 ramillas, para determinar la mortalidad sobre 1000 individuos por repetición. Se concluyó que pyriproxyfen al 0,06\% y buprofezin al $0,1 \%$ aplicados solos, controlan parcialmente los estados juveniles de este insecto en esta época. Además, buprofezin $0,1 \%$ mezclado con aceite mineral $1,5 \%$ controla efectivamente los juveniles de $D$. perniciosus en manzanos y su efecto es similar a la aplicación de aceite mineral $2,0 \%$. Así mismo, la mezcla de pyriproxyfen más aceite mineral 1,5\%, ejerce un control mayor que un tratamiento de aceite mineral $2,0 \%$.

Palabras clave: reguladores de crecimiento, escama de San José, otoño.

\section{ABSTRACT}

Diaspidiotus perniciosus is a major pest of pome and stone fruits that can be controlled using post-harvest applications. The objective of this study was to determine the effect of pyriproxyfen and buprofezin on crawlers (insects in their juvenile stage growth) of $D$. perniciosus (black-cap and grey-cap) on apples var. Royal Gala. The experiment was conducted in the fall of 2012. Insecticides were applied alone and in mixture with mineral oil at $2 \%$ and a control treatment applied only with water. Applications were made with a handgun at 250 PSI and $3500 \mathrm{~L} \mathrm{ha}^{-1}$ over infested apple trees. Crawlers on twigs were evaluated in the laboratory using a stereoscopic magnifier 48 days after application (DAA). The results were expressed in mortality percentage. A completely randomized 
experimental design was used with 6 treatments and 4 replications. The experimental unit consisted of 2 adjacent trees; a sample of 30 twigs was collected from the trees to determine the mortality over 1000 specimens per replication. It was concluded that, when applied alone, pyriproxyfen at $0.06 \%$ and buprofezin at $\mathbf{0 . 1} \%$ can partially control crawlers of $D$. perniciosus in fall. It was also found that buprofezin at $0.1 \%$ in mixture with mineral oil $1.5 \%$ can effectively control San Jose scale crawlers on apple trees, with similar effects to those observed with mineral oil at $2.0 \%$. In addition, the mixture of pyriproxyfen and mineral oil at $1.5 \%$ controls crawlers better than the treatment with mineral oil at $2.0 \%$.

Key words: growth regulators, San Jose scale, fall

\section{INTRODUCCIÓN}

Chile es uno de los principales productores y exportadores de manzanas a nivel mundial, ubicándose en el quinto lugar dentro de los países exportadores, y el segundo generador de divisas en la fruticultura chilena en el 2015 (Muñoz, 2015).

Por otra parte, en los últimos 5 años, la Escama de San José Diaspidiotus perniciosus Comstock (Hemiptera: Diaspididae), se ha convertido en la tercera causal de rechazo en las exportaciones de fruta fresca por presencia en las inspecciones fitosanitarias (Seguel, 2012).

D. perniciosus desarrolla su ciclo de vida principalmente sobre la madera, y por ello una de las formas de diseminación es a través de plantas de vivero. Sin embargo, la forma más efectiva de dispersión es a través del viento. En efecto, especies arbóreas infestadas ubicadas en posición favorable del viento constituyen una fuente permanente de infestación de la plaga, cuyas ninfas pueden desplazarse por el viento decenas de metros desde la fuente de origen (Sazo, 2014).

En manzanas, la mayor presencia se encuentra en la cavidad peduncular y calicinal, produciendo una aureola rojiza entorno al punto de fijación de las ninfas (Welty, 2009). Por otra parte, a nivel de "packing", la presencia de este insecto genera costos mayores por cuanto se reduce la eficiencia en el proceso de selección y embalaje (Seguel, 2012).

En Chile esta especie en la zona central presenta tres generaciones durante la temporada; el primer nacimiento de ninfas ocurre entre fines de octubre y comienzos de noviembre y se prolonga por cuatro a seis semanas; el segundo ocurre a partir de la segunda semana de enero y el tercero durante el mes de marzo, prolongándose incluso hasta fines de abril en condiciones extraordinarias (Sazo y Campos, 1986).

El control de D. perniciosus se realiza mediante el empleo de insecticidas, puesto que el control natural en Chile no es efectivo en un sistema productivo intervenido (Sazo, 2011). La época más efectiva para el control de esta plaga es a finales del invierno (Charlin y Sazo, 1988).

La estrategia de control consiste en el uso del aceite mineral aplicado solo o mezclado con insecticidas en invierno y se complementa en primavera y/o verano (Sazo, 2011), con insecticidas organofosforados sintéticos (clorpirifos, diazinon o metidation) (Sazo y Campos, 1986; Alston et al., 2011) o neonicotinoides (acetamiprid) o sulfoxaminas (sulfoxaflor) o ácidos tetrónicos (spirotetramato) dirigido a las ninfas móviles de la primera o segunda generación de la temporada (Welty, 2009; Besin, 2010; Alston et al., 2011).

A mediados de la primera década del presente siglo se detectó en Chile resistencia de D. perniciosus a clorpirifos en ciruelos y manzanos, cuyos factores de resistencia fueron 32,3 y 25,1 , respectivamente; este fenómeno se extendió rápidamente en estas especies frutales afectando la producción y exportación por rechazo en las inspecciones fitosanitarias (Cañas, 2010). Este hecho produjo una reducción en el uso de insecticidas organoforados y su remplazo por insecticidas neonicotinoides y reguladores de crecimiento (IRC) como pyriproxyfen (Eddleston et al. 2008), el cual posee un modo de acción diferente, además de una alta selectividad sobre especies que no son objeto de control (Lobos, 2010).

En ensayos realizados con acetamiprid, clorpirifos, phosmet y pyriproxyfen sobre D. perniciosus, se evidenció que pyriproxifen aplicado en manzano bajo condiciones de campo, limita el desarrollo de las ninfas una vez establecidas (Sanhueza, 2011). Así mismo, Sazo et al. (2008), mencionan que aplicaciones de pyriproxifen dirigida a ninfas de primera generación de $D$. perniciosus en manzano, reduce el número de ninfas fijadas en ramillas y su efecto es similar al obtenido con aplicaciones de clorpirifos. Pyriproxifen ha mostrado ejercer un buen control sobre D. perniciosus, con resultados similares a aplicaciones con aceite mineral sobre ninfas móviles (Beers y Himmel, 2002).

El objetivo de este estudio fue evaluar el efecto insecticida de buprofezin y pyriproxifen aplicados solos y en mezcla con aceite mineral sobre $D$. perniciosus en manzanos var. Royal Gala en postcosecha. 


\section{MATERIALES Y MÉTODOS}

El estudio se realizó en un huerto de manzanos variedad Royal Gala plantado a $4 \times 3 \mathrm{~m}$, infestado con D. perniciosus, ubicado en el sector "El Olivar"

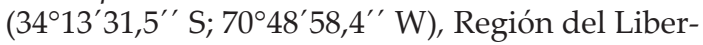
tador Bernardo O'Higgins, Chile, en mayo del 2012, una vez concluido el nacimiento de ninfas de la tercera generación de la temporada.

Se estudió pyriproxyfen (Admiral ${ }^{\circledR} 10$ EC), buprofezin (Applaud ${ }^{\circledR} 25 \mathrm{WP}$ ), aceite mineral (Elf purespray ${ }^{\circledR} 15 \mathrm{E}$ ) y un testigo aplicado con agua; las concentraciones analizadas se presentan en la Tabla 1. Se marcaron 48 árboles con un nivel de infestación similar y luego de sortear los tratamientos, se realizaron las aplicaciones mediante una motopulverizadora (Levera, Arrastre de 220 L, Santiago, Chile), equipada con una bomba de membrana Comet de $40 \mathrm{~L} \mathrm{~min}^{-1}$, pitón, 250 psi y 4,2 L planta ${ }^{-1}$ (3500 L ha-1).

La evaluación se realizó 48 días después de la aplicación, sobre una población de 1000 individuos (gorrita negra-gris) provenientes de una muestra compuesta de 30 ramillas por unidad experimental, colectadas en la parte media del árbol. Se consideró insecto vivo aquel que al levantar el escudo mostraba el cuerpo turgente y color amarillo-limón; el resto se consideró muerto.

Se empleó diseño de aleatorización completa con seis tratamientos y cuatro repeticiones. La unidad experimental fueron dos árboles contiguos y la unidad muestral fue 30 ramillas.

Los resultados expresados en porcentajes de mortalidad de ninfas se normalizaron mediante la transformación angular de Bliss y se sometieron al análisis de varianza (ANDEVA) y Prueba de rango múltiple de Duncan, para separación de medias, con un nivel de confianza del $95 \%$.

\section{RESULTADOS Y DISCUSIÓN}

Los resultados de mortalidad de estados juveniles de $D$. perniciosus expresados en porcentaje se presentan en la Tabla 2, donde se observa que existen diferencias estadísticamente significativas entre los tratamientos evaluados según el análisis

Tabla 1. Tratamientos en ensayo con buprofezin y pyriproxifen para control de Escama de San José (D. perniciosus)-

Table 1. Treatments used in the trials with buprofezin and pyriproxyfen to control San Jose scale ( $D$. perniciosus)

\begin{tabular}{lc}
\hline \multicolumn{1}{c}{ Ingrediente activo } & Concentración \\
\hline & $\%$ \\
Testigo & - \\
Aceite mineral & 2 \\
Buprofezin & 0,1 \\
Buprofezin + Aceite mineral & $0,1 \%+1,5$ \\
Pyriproxyfen & 0,06 \\
Pyriproxyfen + Aceite mineral & $0,06+1,5$ \\
\hline
\end{tabular}

Tabla 2. Mortalidad (\%) de estados juveniles de D. perniciosus, fijados en ramillas de la temporada 48 DDA de pyriproxifen, buprofezin y aceite mineral en postcosecha.

Table 2. Mortality ( $\%$ ) of crawlers of $D$. perniciosus on twigs 48 days after application of pyriproxyfen, buprofezin and mineral oil at post-harvest time.

\begin{tabular}{lc}
\hline Tratamiento & Mortalidad \\
\hline & $\%$ \\
Testigo & $27,45 \mathrm{a}$ \\
Buprofezin $(0,1 \%)$ & $50,98 \mathrm{~b}$ \\
Pyriproxyfen $(0,06 \%)$ & $54,20 \mathrm{~b}$ \\
Aceite mineral $(2 \%)$ & $87,33 \mathrm{c}$ \\
Buprofezin + Aceite mineral $(0,1 \%+1,5 \%)$ & $91,68 \mathrm{~cd}$ \\
Pyriproxyfen + Aceite mineral $(0,06 \%+1,5 \%)$ & $95,00 \mathrm{~d}$ \\
\hline
\end{tabular}

DDA: días después de la aplicación.

Promedios con letras diferentes en la columna indican diferencias estadísticamente significativas entre los tratamientos según el test de Duncan $(P \leq 0,05)$. 
de comparaciones múltiples, estableciendo cuatro niveles de mortalidad.

El tratamiento testigo expresa diferencias estadísticamente significativas con el resto de los tratamientos, con un porcentaje de mortalidad del $27,45 \%$, considerado bajo, lo que se asocia a temperaturas moderadas en la zona central de Chile, concordando con lo reportado por González (1981) con un porcentaje de mortalidad del $18,7 \%$, Pless et al. (1995) con un 25\%, y Lurie et al. (1998) con un $34 \%$.

Los insecticidas reguladores de crecimiento buprofezin $(50,98 \%)$ y pyriproxyfen $(54,20 \%)$ aplicados solos, presentan un nivel de mortalidad intermedio, demostrando que a pesar de la diferencia específica en sus mecanismos de acción, no reflejan una diferencia estadísticamente significativa entre sí. Sin embargo, fueron estadísticamente distintos al resto de tratamientos insecticidas.

El aceite mineral al $2 \%$ presentó un control efectivo de los estados juveniles de la escama de San José, y su efecto fue similar estadísticamente a buprofezin $0,1 \%$ + aceite mineral $1,5 \%$. Pyriproxyfen $0,06 \%$ + aceite mineral al $1,5 \%$ no presentó diferencias significativas respecto a buprofezin $0,1 \%$ + aceite mineral $1,5 \%$, sin embargo, fue diferente a aceite mineral al $2 \%$.

En relación a los insecticidas reguladores de crecimiento y aceite mineral, Sazo et al. (2008) señalan que en bajos niveles de infestación de esta plaga, una aplicación de aceite mineral o pyriproxyfen logran reducir el número de ninfas fijadas en ramillas, obteniendo un efecto similar a una aplicación de clorpirifos en primavera.

Así mismo, Bentley et al. (2000a) mencionan que aplicaciones de aceite mineral, buprofezin y pyriproxyfen ejercen un control eficiente en $D$. perniciosus sobre ciruelo. En tanto, Bently et al. (2000b) indican que aplicaciones de aceite mineral en ciruelos con una baja infestación, controlan adecuadamente $D$. perniciosus sin la necesidad de complementar con insecticidas organofosforados sintéticos o reguladores de crecimiento en primavera.

Por otra parte, Langley et al. (1990) indicaron que aplicaciones de aceite de canola (Brassica napus L.) con pyriproxyfen aumenta la vulnerabilidad de la mosca tsetse (Glossina morsitans), estableciendo que el aceite puede facilitar la penetración de este regulador de crecimiento a través de la cutícula del insecto. Estos resultados coinciden con lo observado en este estudio, por cuanto la mezcla de reguladores de crecimiento (buprofezin y pyriproxyfen) más aceite mineral mostró mayor eficacia que la aplicación de ambos reguladores solos.

Finalmente, cabe señalar que tanto buprofezin como pyriproxyfen evidenciaron un nivel de control similar, lo que podría ser una alternativa válida para ser aplicados en forma alternada en manzanos, puesto que poseen diferentes modos de acción. Así mismo, D. perniciosus no ha desarrollado resistencia a estos reguladores de crecimiento, sin embargo, para la escama roja de California (Aonidiella aurantii), se ha reportado una resistencia incipiente a pyriproxyfen (Eliahu et al., 2007).

\section{CONCLUSIONES}

Aplicaciones de buprofezin al $0,1 \%$ y pyriproxyfen al $0,06 \%$ sobre estados juveniles de $D$. perniciosus en manzanos en otoño, ejercen un control parcial de la plaga. Las mezclas de buprofezin y pyriproxyfen con aceite mineral 1,5\% controlan en forma efectiva esta plaga, sin embargo, la mezcla de buprofezin con aceite mineral 1,5\% ejerce un control similar a las aplicaciones de aceite mineral $2 \%$. Finalmente, la mezcla de pyriproxyfen con aceite mineral 1,5\% presenta un mayor control de D. perniciosus que aceite mineral $2 \%$.

\section{LITERATURA CITADA}

Alston, D., M. Murray, and M. Reding. 2011. San Jose Scale (Quadraspidiotus perniciosus). (ENT153-06), Utah State University Extension and Utah Plant Pest Diagnostic Laboratory. Utah, U.S.A. Available at http://extension.usu. edu/files/publications/publication/san-josescale'97.pdf (Accessed 3 March 2015).

Beers, E., and P. Himmel. 2002. Effect of chloronicotinyl insecticides on phytophagous and predatory mite populations in a cover spray program. $\mathrm{p}$. 16. In Proceeding 76th Annual Western Orchard Pest \& Disease Management Conference. 9-11 January. Portland, Oregon, U.S.A. Available at http://entomology.tfrec. wsu.edu/wopdmc/2002PDFs/Rep02\%20 Chemical\%20Beers5.pdf (Accessed 3 March 2015).

Bentley, W., D. Rice, K. Day, and C. Hernandez. 2000a. Managing San Jose Scale with dormant oils. Plant Protection Quarterly 10(2):1-4.

Bentley, W., L. Martin, D. Rice, B. Ribiero, and K. Day. 2000b. Further investigation in the management of San Jose Scale with narrow range horticultural oil. Plant Protection Quarterly 10(4): 5-6.

Besin, R. 2010. San José Scale. (Entfact-204). Cooperative Extension Service, College of Agriculture, University of Kentucky. Kentucky, USA. Available at http://www.ca.uky.edu/ entomology/entfacts/ef204.asp (Accessed 13 June 2015). 
Cañas, B. 2010. Detección de la resistencia de Diaspidiotus perniciosus a clorpirifos en frutales. Tesis Ingeniero Agrónomo. Facultad de Ciencias Agronómicas, Universidad de Chile, Santiago, Chile.

Charlin, R., y L. Sazo. 1988. Comparación etológica y morfológica de la escama del acacio y alamo Diaspidiotus ancyclus (Putnam) y la escama de San José Quadraspidiotus perniciosus (Comst.). Revista Aconex 22:21-24.

Eddleston M., N. Buckley, P. Eyer, and A. Dawson. 2008. Management of acute organophosphorus pesticide poisoning. Lancet 371(9612):597-607.

Eliahu, M., D. Blumberg, A. Horowitz, and I. Ishaaya. 2007. Effect of pyriproxyfen on developing stage and embryogenesis of California Red Scale (CRS), Aonidiella aurantii. Pest Management Science 73:743-746.

González, R. 1981. Biología, ecología y control de la escama de San José en Chile: Quadraspidiotus perniciosus (Comst). Universidad de Chile, Facultad de Ciencias Agronómicas, Santiago, Chile.

Langley, P., T. Felton, K. Stafford, and H. Oochi. 1990. Formulation of pyriproxyfen, a juvenile hormone mimic, for tsetse control. Medical and Veterinary Entomology 4(2):127-133.

Lobos, E. 2010. Insecticidas reguladores de crecimiento (IGR's). Consideraciones para su uso en la protección de cultivos. Sitio Argentino de Producción Animal. Disponible en http:// www.produccion-animal.com.ar/produccion_y_manejo_pasturas/pasturas_combate_ de_plagas_y_malezas/119-reguladores_crecimiento.pdf (Consulta 12 mayo 2015)

Lurie, S., E. Fallik, J. Klein, F. Kozar, and K. Kovac. 1998. Postharvest heat treatment of apples to control of San Jose Scale (Quadraspidiotus perniciosus Comstock) and blue mold (Penicillium expansum Link) and maintain fruit firmness. HortScience 123(1):110-114.

Muñoz, M. 2015. Boletín frutícola: Avance marzo 2015. Oficina de Estudios y Precios Agrícolas (ODEPA). Santiago, Chile. Disponible en http://www.odepa.cl/wp-content/files_mf/ 1431097846Boletinfrut\%C3\%ADcola2015042. pdf (Consulta 04 de Abril 2015)
Pless, C., D. Deyton, and C. Sams. 1995. Control of San Jose Scale, and European Red Mite on dormant fruit trees with soybean oil. HortScience 30(1):94-97.

Sanhueza, V. 2011. Efecto residual de acetamiprid, phosmet, clorpirifos y pyriproxifen sobre escama de San José en manzanas. Tesis Ingeniero Agrónomo. Facultad de Ciencias Agronómicas, Universidad de Chile, Santiago, Chile.

Sazo, L. 2011. Se debe actuar pensando que la Escama está presente en todos los huertos. Red Agrícola. $\mathrm{N}^{\circ}$ 42. Disponible en http://www. redagricola.com/reportajes/fitosanidad/sedebe-actuar-pensando-que-la-escama-estapresente-en-todos-los-huertos (Consulta 22 junio 2012).

Sazo, L. 2014. Scale, San Jose scale. 188-189. In Sutton, T. H. Aldwinckle, A. Agnello, J. Walgenbach (eds.). Compendium of apple and pear diseases and pests. $2^{\text {nd }}$. ed. The American Phytopathological Society, St. Paul, Minnesota, U.S.A.

Sazo, L., y L. Campos. 1986. Reconocimiento, desarrollo y control de escama de San José en Chile. Revista Aconex 13:15-21.

Sazo, L., J. Araya, and S. Esparza. 2008. Control of San José Scale nymphs, Diaspidiotus perniciosus (Comstock), on almond and apple orchards with pyriproxyfen, phenoxycarb, chlorpyriphos, and mineral oil. Chilean Journal of Agricultural Research 68(3):284-289.

Seguel, P. 2012. Escama de San José (Diaspidiotus perniciosus): Grave problema en huertos, procesos y exportación. Revista Frutícola 1:2325.

Welty, C. 2009. San José Scale on fruit trees. (HYG 2039-09). Agriculture and Natural Resources, Department of Entomology, The Ohio State University Extension, Ohio, U.S.A. Available at http://ohioline.osu.edu/hyg-fact/2000 pdf/2039.pdf (Accessed 22 June 2015). 